\title{
Strates
}

STRATES Matériaux pour la recherche en sciences sociales

$10 \mid 2001$

Villageois et citadins de Grèce

\section{L'Albanie : un exemple sensible du maillage européen}

Marie-Alix Carlander

\section{(2) OpenEdition}

1 Journals

Édition électronique

URL : http://journals.openedition.org/strates/385

DOI : $10.4000 /$ strates.385

ISSN : $1777-5442$

Éditeur

Laboratoire Ladyss

Édition imprimée

Date de publication : 1 mai 2001

ISSN : 0768-8067

Référence électronique

Marie-Alix Carlander, «L'Albanie : un exemple sensible du maillage européen », Strates [En ligne], 10 |

2001, mis en ligne le 12 janvier 2005, consulté le 25 septembre 2020. URL : http://

journals.openedition.org/strates/385; DOI : https://doi.org/10.4000/strates.385

Ce document a été généré automatiquement le 25 septembre 2020.

Tous droits réservés 


\title{
L'Albanie : un exemple sensible du maillage européen
}

\author{
Marie-Alix Carlander
}

\section{Artan FUGA}

Identités périphériques en Albanie (La recomposition du milieu rural et les nouvelles dynamiques politiques)

L'Harmattan, 2000, 288 pages

Le livre d'Artan Fuga, chercheur associé et professeur invité au laboratoire Ladyss (Cnrs, Université Paris X-Nanterre), qui vient de paraître chez l'Harmattan, analyse les deux dynamiques de la transformation post-communiste en Albanie: la réforme économique avec ses conséquences sociales et les changements politiques, en particulier les variations du comportement électoral de la société albanaise. L'aspect économique et social et l'aspect politique et institutionnel sont liés et subissent des influences réciproques dans le cadre d'une dialectique que le lecteur peut saisir au fur et à mesure des interprétations effectuées dans le livre. L'auteur privilégie l'étude du milieu rural en Albanie sachant que ce pays a une forte densité de population rurale, que son économie est largement fondée sur la production agricole et que l'exode rural a affecté sensiblement la vie urbaine et les rapports entre les villes et les campagnes. La vie économique et sociale d'une société fonctionnant comme un tout, chaque dynamique partielle s'inscrit dans un contexte social général. Artan Fuga, dans son livre, respecte ce principe méthodologique et nous présente aussi en détails les transformations économiques dans la zone urbaine.

5 En ce qui concerne la réforme économique, l'auteur nous offre plusieurs concepts indispensables pour comprendre la réalité concrète du pays. Il fait la distinction entre des notions comme, par exemple, la privatisation, la re-privatisation, la restitution des biens aux anciens propriétaires, etc., et approfondit son analyse concernant les deux principales logiques qui ont conduit la transition économique albanaise. La première est fondée sur le principe du droit historique qui privilégie les anciens propriétaires fonciers ou autres, la deuxième donne la priorité absolue au principe du droit actuel, c'est-à-dire, fait avantager les personnes et les groupes sociaux qui utilisaient les 
moyens de productions au moment de l'application des réformes commencées à large échelle à partir de 1992. Selon l'auteur, la vie sociale et politique conflictuelle qu'a connu le pays, certains dysfonctionnements qui bloquent même actuellement la reprise économique, plusieurs phénomènes sociaux négatifs, prennent racines précisément dans le conflit des logiques transformatrices appliquées par les pouvoirs locaux et par les agences internationales.

6 Dans ce livre, le lecteur peut constater la fidélité de l'auteur vis-à-vis d'une méthodologie plurifactorielle concernant la présentation du sujet. La réforme économique et le comportement électoral dont émanent les institutions politiques du pays ne sont pas le fruit du hasard. Ils sont déterminés par un ensemble de facteurs sociaux, psychologiques, de propagande, traditionnels, culturels, religieux, qui ont été largement pris en considération tout au long des analyses entreprises. Ainsi, Artan Fuga utilise des chiffres et des données empiriques riches afin d'argumenter ses thèses sur la réforme économique, entreprend une longue analyse des principaux groupes sociaux en tant que véritables acteurs de la vie sociale, décrit des modes de vie et d'habiter, surtout dans les zones rurales du pays, fait des comparaisons entre diverses zones géographiques et sociales du pays et entre l'état actuel de l'économie albanaise et l'ancien modèle d'économie socialiste, analyse les représentations collectives appartenant à diverses couches de la population albanaise et complète ses outils méthodologiques en se basant aussi sur des résultats de divers sondages réalisés par des chercheurs albanais et étrangers sur l'opinion publique en Albanie.

7 L'auteur utilise un vaste univers conceptuel pour caractériser les différentes phases de la dynamique politique du pays et le comportement politique du citoyen albanais face aux changements sociaux qui ont eu lieu durant la phase de la transition. Il parle d'une culture politique mixte qui met ensemble des tendances sociales et politiques nourries au sein de la société rurale et de la culture citadine. Le principal point de contact entre les villes et les campagnes est actuellement l'exode rural, phénomène massif qui bouleverse la société albanaise. L'auteur étudie le rythme de cet exode, les lignes principales de son orientation géographique, analyse les conséquences économiques et sociales de ce phénomène sur la stabilité politique et économique du pays et l'impact qu'il a sur la vie des familles albanaises.

8 En tant que philosophe, Artan Fuga reprend certains concepts élaborés par la pensée philosophique européenne et américaine en les utilisant comme lignes directrices pour ses propres réflexions. Il commence son livre en présentant tout d'abord le concept du temps politique cyclique, il reprend des idées concernant le rapport entre l'ouverture, la mondialisation et la modernisation et il s'engage dans un débat concernant la « nature politique » du paysan.

9 L'auteur considère que le cas albanais répond bien à une dynamique qu'il désigne comme " périphérique » par rapport à l'Union européenne. Ainsi, la société albanaise, une fois entrée en contact avec l'Europe occidentale, ne peut être étudiée qu'à travers cette interaction qui met en contact des réalités sociales, économiques et culturelles différentes. L'Albanie subit une déstructuration des anciens liens sociaux, liée à une certaine ouverture vis-à-vis

de l'Europe et du monde, et, d'autre part, elle en

11 bénéficie sous forme de technologie, d'infrastructure communicationnelle, de services, etc. De toute façon, l'inondation du marché albanais par des produits étrangers provenant d'Italie et de Grèce crée des difficultés presque insurmontables pour le 
fonctionnement de l'économie locale. Les grandes différences entre le rapport travail/ salaire en Albanie et dans les pays européens voisins incitent la main-d'œuvre albanaise à partir en Occident et casse l'envie de travailler de ceux qui restent au pays. Ainsi, un remaniement du modèle économique du pays dans un contexte international s'impose.

L'auteur termine ses réflexions en posant une question essentielle concernant l'identité sociale, économique et culturelle que doit avoir la zone des pays balkaniques mis en contact, d'une façon récente, avec l'Union européenne et manifestant l'envie de s'y associer à travers une stratégie à long terme. Au lieu d'une identité homogénéisée et homogénéisante qui perçoive automatiquement la périphérie comme une réalité prête à se construire selon les logiques qui fonctionnent au centre, l'auteur nous présente le concept d'une "identité différentielle " qui, en respectant les différences des pays avoisinant l'Union européenne, servirait à y implanter des structures qui permettent le bon fonctionnement de la société et feraient naître des besoins fonctionnels relatifs à une perspective d'intégration européenne.

13 Le livre d'Artan Fuga par son thème et la problématique qu'il met en exergue, les interprétations proposées, l'information qu'il apporte, les perspectives futures de la zone qu'il cherche à questionner, les thèses et les hypothèses qu'il met en circulation, représente un travail essentiel pour nous faire comprendre toujours mieux la réalité actuelle des pays de l'Est durant leur phase post-communiste. Venant après son premier livre L'Albanie entre la pensée totalitaire et la raison fragmentaire, publié aussi chez l'Harmattan, cet ouvrage poursuit incontestablement une recherche réussie en nous proposant, à partir du cas concret de l'Albanie, une analyse fondée philosophiquement de la transformation d'un pays à l'identité bien définie, structurellement bloqué et fermé, à un pays potentiellement ouvert et souple. 